Critical phenomena in neutron stars: I. Linearly unstable nonrotating models

This article has been downloaded from IOPscience. Please scroll down to see the full text article.

2010 Class. Quantum Grav. 27235015

(http://iopscience.iop.org/0264-9381/27/23/235015)

View the table of contents for this issue, or go to the journal homepage for more

Download details:

IP Address: 194.94.224.254

The article was downloaded on 16/04/2013 at 08:16

Please note that terms and conditions apply. 


\title{
Critical phenomena in neutron stars: I. Linearly unstable nonrotating models
}

\author{
David Radice $^{1}$, Luciano Rezzolla ${ }^{1,2}$ and Thorsten Kellerman ${ }^{1}$ \\ 1 Max-Planck-Institut für Gravitationsphysik, Albert Einstein Institut, Potsdam, Germany \\ 2 Department of Physics and Astronomy, Louisiana State University, Baton Rouge, LA, USA \\ E-mail: kellerma@aei.mpg.de
}

Received 16 July 2010, in final form 21 September 2010

Published 12 November 2010

Online at stacks.iop.org/CQG/27/235015

\begin{abstract}
We consider the evolution in full general relativity of a family of linearly unstable isolated spherical neutron stars under the effects of very small perturbations as induced by the truncation error. Using a simple idealfluid equation of state, we find that this system exhibits a type I critical behaviour, thus confirming the conclusions reached by Liebling et al (2010 arXiv:1001.0575v1) for rotating magnetized stars. Exploiting the relative simplicity of our system, we are able to carry out a more in-depth study providing solid evidence of the criticality of this phenomenon and also to give a simple interpretation of the putative critical solution as a spherical solution with the unstable mode being the fundamental F-mode. Hence for any choice of the polytropic constant, the critical solution will distinguish the set of subcritical models migrating to the stable branch of the models of equilibrium from the set of subcritical models collapsing to a black hole. Finally, we study how the dynamics changes when the numerical perturbation is replaced by a finitesize, resolution-independent velocity perturbation and show that in such cases a nearly critical solution can be changed into either a sub- or supercritical one. The work reported here also lays the basis for the analysis carried in a companion paper, where the critical behaviour in the head-on collision of two neutron stars is instead considered (Kellerman et al 2010 Class. Quantum Grav. 27 235016).
\end{abstract}

PACS numbers: 04.25.Dm, 04.40.Dg, 04.70.Bw, 95.30.Lz, 97.60.Jd

\section{Introduction}

Critical phenomena in general relativity were first discovered by Choptuik [3] for the gravitational collapse of a massless scalar field. After his seminal work, these phenomena were also discovered for a wide range of systems, including massive scalar fields and ultrarelativistic fluids [4-12] (see also [13] for a recent review). Our interest here is to further 
develop the analysis of critical phenomena in the presence of (perfect) fluids in general and of relativistic spherical stars in particular.

We recall that the first simulations of critical collapse of a perfect fluid were performed by Evans and Coleman [4]. They used an ultra-relativistic equation of state (EOS) with polytropic exponent $\Gamma=4 / 3$ (radiation) and found evidence of a continuously self-similar (CSS) critical solution. Following works by [5] and [14] showed that type II critical phenomena occur for every value of $\Gamma$ between 1 and 2 with a CSS critical solution. The ultra-relativistic EOS, that is one in which the pressure is proportional to the energy density $p=(\Gamma-1) e$, is the only scale-invariant EOS in general relativity, thus the only one which is compatible with the existence of a homothetic vector field. In other words, fluids obeying an ultra-relativistic EOS are the ones that admit self-similar solutions in general relativity [15]. Nevertheless, evidence of CSS critical solutions was found by Neilsen and Choptuik [6] also for perfect fluids with an ideal-gas EOS. They conjectured that, as type II phenomena are kinetically dominated, an ideal gas would behave like an ultra-relativistic gas and thus nearly critical solutions will approach the corresponding ultra-relativistic CSS solution. They were actually able to show that this is indeed the case by comparing ultra-relativistic CSS solutions, obtained using the self-similarity ansatz, to the corresponding ideal-gas solution. This suggested the possibility of observing type II critical phenomena in the context of neutron star (NR) collapse.

The first studies in this direction were performed by Novak [7]. He studied solutions with velocity-induced perturbation of a Tolman-Oppenheimer-Volkoff (TOV) background and found a type II critical phenomenon using a stiff ideal-gas EOS (i.e. with $\Gamma=2$ ) and even a more realistic tabulated EOS. Noble [8] studied critical phenomena in NR collapse in great detail by using the gravitational interaction with a massless scalar field to perturb spherical-star configurations. He confirmed the observation by Novak that a minimum mass is required to trigger collapse and also found that, for very high mass spherical stars, a type I critical phenomenon is observed with each model oscillating around a corresponding solution on the unstable branch. More recently, Noble and Choptuik [9] performed an accurate study using the same setup of [7] and were able to show that, even for initial data of spherical stars, the scaling exponent in the mass relation law is compatible with the exponent in the corresponding ultra-relativistic case.

Type I critical phenomena in NR collapse have seen a renewed interested mainly due to the discovery by Jin [10] of critical phenomena in the head-on collision of NS. They considered the families of two equal mass NS, modelled with an ideal-gas EOS, boosted towards each other at a prescribed speed and varied the mass of the stars, their separation, velocity and $\Gamma$ parameter. They found that at the threshold of black-hole formation, a type I critical phenomenon can be observed, with the putative solution being an oscillating star. In a successive paper, Wan et al [16] repeated the same results by performing the head-on collision of Gaussian packets. They also claimed that the critical solution is a new kind of metastable object and not a perturbed spherical star as its mass is less than the maximum allowed mass of a spherical star for the same EOS [10] and its oscillation frequencies are one order of magnitude larger than the eigenfrequencies of a spherical star with the same total baryonic mass [16]. These claims have partly been rejected by the work carried out in [2], which also investigated critical phenomena in the head-on collision of equal-mass spherical stars and found that the critical solution can be compared to a spherical star solution sitting on the unstable branch.

Motivated by these recent results, we have investigated phase transitions in the families of linearly unstable spherical stars, a case substantially unexplored in previous systematic works. We show that this transition is a critical one and that the putative critical solution can be interpreted as an oscillating spherical star. We further study the effects of the introduction of velocity-induced perturbations, as the ones used by [7], on nearly critical solutions. 
The remainder of this paper is organized as follows. In section 2 we give a brief introduction to critical phenomena in general relativity. In section 3 we describe the numerical settings of the simulations and the properties of the used initial data. In section 4 we show in detail our results, while in section 5 we give the conclusions and discussion. We use a spacetime signature $(-,+,+,+)$, with Greek indices running from 0 to 3 and Latin indices from 1 to 3 . We also employ the standard convention for the summation over repeated indices. Unless otherwise stated, all the quantities are expressed in a system of units in which $c=G=M_{\odot}=1$.

\section{Critical phenomena in gravitational collapse}

In what follows we give a brief overview of critical phenomena in gravitational collapse which will be useful to cast our results in the more general context of critical phenomena in general relativity. We refer the interested reader to [13] for a more systematic presentation.

\subsection{Self-similarity}

Before dwelling on critical phenomena and because self-similarity plays a central role in this context, it is useful to recall briefly the definitions of 'continuous' self-similarity and 'discrete' self-similarity. We refer the interested reader to [17] for a more detailed discussion.

We recall that a spacetime is said to be continuously self-similar if there exists a vector field, $\xi^{\mu}$, such that $\nabla_{(\mu} \xi_{v)}=g_{\mu \nu}$. Vector fields satisfying this condition are said to be 'homothetic' as we can easily construct a one-parameter group of transformations, $\phi_{s}: x^{\mu} \mapsto y^{\mu}(s)$, where $y^{\mu}(s)$ is the integral curve associated with $\xi^{\mu}$ passing through $x^{\mu}$. It is then easy to see that $\phi_{s}$ is a homothetic transformation as the associated push-forward acts as a rescaling on the metric

$$
\phi_{s}^{*} g_{\mu \nu}=\mathrm{e}^{2 s} g_{\mu \nu} .
$$

For this reason in a system of coordinates adapted to the self-similarity

$$
\xi^{\mu}=-\left(\frac{\partial}{\partial \tau}\right)^{\mu}
$$

the metric coefficients read

$$
g_{\mu \nu}\left(\tau, x^{i}\right)=\mathrm{e}^{-2 \tau} \tilde{g}_{\mu \nu}\left(x^{i}\right),
$$

and the new metric $\tilde{g}_{\mu \nu}$ appears explicitly self-similar, i.e. independent of $\tau$.

Similarly, a spacetime is said to be 'discretely self-similar' (DSS) if a discrete version of equation (1) holds. In particular, Gundlach [18] defines a spacetime to be DSS if there exists a diffeomorphism $\phi$ and a real constant $\Delta$ such that for any positive integer $n$,

$$
\left(\phi^{*}\right)^{n} g_{\mu \nu}=\mathrm{e}^{2 n \Delta} g_{\mu \nu} .
$$

In coordinates adapted to the self-similarity, a point $P$ with coordinates $\left(\tau, x^{i}\right)$ is mapped by $\phi$ onto $\left(\tau-\Delta, x^{i}\right)$ and the metric can be written as

$$
g_{\mu \nu}\left(\tau, x^{i}\right)=\mathrm{e}^{-2 \tau} \tilde{g}_{\mu \nu}\left(\tau, x^{i}\right),
$$

where

$$
\tilde{g}_{\mu \nu}\left(\tau+\Delta, x^{i}\right)=\tilde{g}_{\mu \nu}\left(\tau, x^{i}\right) .
$$

Thus, if $\nabla^{\mu} \tau$ is timelike and induces a Cauchy foliation of the spacetime, we can give a physical interpretation of the dynamics of DSS solutions as a combined effect of rescaling and a periodic 'echoing' of the geometry. 


\subsection{The basic concepts}

Let us consider a group of one-parameter families of solutions, $\mathcal{S}[P]$, of the Einstein equations such that for every $P>P^{\star}, \mathcal{S}[P]$ contains a black hole and for every $P<P^{\star}, \mathcal{S}[P]$ is a solution not containing singularities. We say that these families exhibit a critical phenomenon if they have the common property that, as $P$ approaches $P^{\star}, \mathcal{S}[P]$ approaches a universal solution $\mathcal{S}\left[P^{\star}\right]$, i.e. not depending on the particular family of initial data, and that all the physical quantities of $\mathcal{S}[P]$ depend only on $\left|P-P^{\star}\right|$. In analogy with critical phase transitions in statistical mechanics, these phenomena are then classified as type II or type I critical phenomena [13]. In what follows we briefly recall the differences between the two classes.

2.2.1. Type II critical phenomena. Type II critical phenomena involve the existence of CSS or DSS solutions sitting at the threshold of black-hole formation. They are characterized by the mass-scaling relation

$$
M_{\mathrm{BH}}=c\left|P-P^{\star}\right|^{\gamma}
$$

where $\gamma$ is independent of the particular choice of the initial data. The nomenclature 'type II' comes from the analogous type II phase transitions in statistical mechanics, which are characterized by scale invariance of the thermodynamical quantities [13].

These phenomena are usually interpreted in terms of attractors in an infinite-dimensional phase space, but we will here present a qualitative picture which can be useful to fix the ideas (see also the review in [13]). A more rigorous study employing the renormalization group formalism can be found instead in [5].

Let us consider general relativity as an infinite-dimensional dynamical system in an abstract phase space in which extra gauge freedoms have been eliminated so that each point, $Z$, can be thought as an initial dataset for the Einstein equations and the associated time development as a line in this space: $t \mapsto Z(t)$. We suppose to have chosen a slicing adapted to the self-similarity of the critical solution so that it appears as a fixed point, $Z^{\star}$, in the CSS case or a closed orbit for the DSS case (see [13] for a more in-depth discussion of the consequences of these assumptions).

In the case of CSS solutions, the main features of this phase space are the presence of two attractive sets: $A$ and $B$ representing regular solutions without singularities and blackhole solutions. Their basins of attractions are separated by a manifold, $\mathcal{C}$, called critical manifold on which there is an attractor of codimension 1: the critical solution, $Z^{\star}$; this is shown schematically in figure 1 . Any generic one-parameter family of initial data can then be thought as a one-dimensional line intersecting the critical manifold in one point. Initial data, with $P<P^{\star}$, will develop as regular solutions not containing singularities and will therefore fall in the basin of attraction containing the so-called subcritical solutions (cf set $A$ in figure 1). Conversely, solutions with $P>P^{\star}$ will undergo gravitational collapse with the formation of a black hole, thus falling in the basin of attraction containing the so-called supercritical solutions (cf set $B$ in figure 1).

The key point here is that the critical solution is attractive on the critical manifold. Stated differently, nearly critical solutions will experience 'funnelling' effects as all but one mode converges towards $Z^{\star}$. If $P \approx P^{\star}$, then the unstable mode, i.e. the mode 'perpendicular' to $\mathcal{C}$, will be small until later in the evolution, thus allowing for the observation of nearly critical solutions. In this case, all but one mode of the solution is 'washed out' by the interaction with the critical solution, thus explaining both the universality of the solution and the mass-scaling relation. 


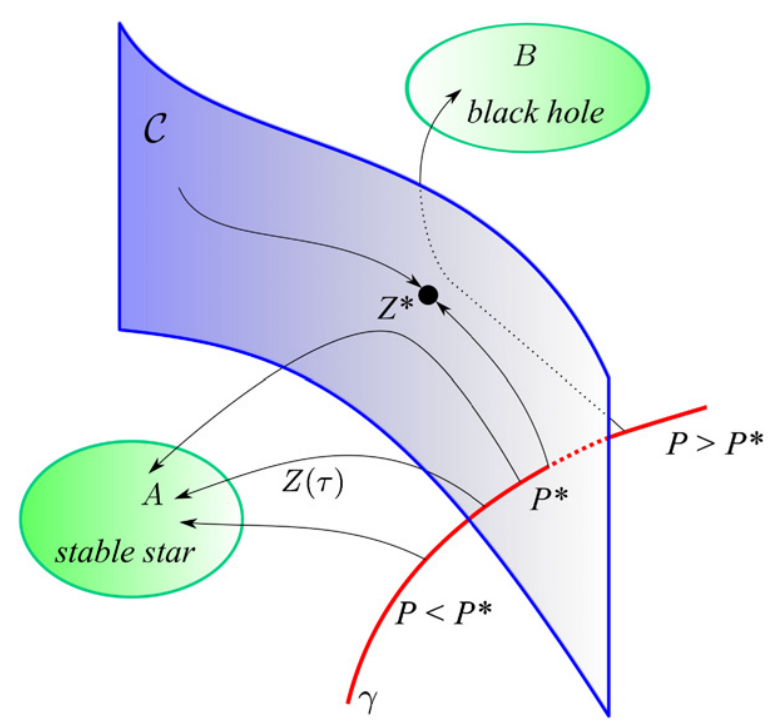

Figure 1. Phase space picture of type II critical phenomena. The surface $\mathcal{C}$ represents the critical manifold, separating the basins of attraction of $A$ and $B$. The line $\gamma$ represents a generic one-parameter family of initial data intersecting the critical manifold in $P^{\star}$. Generic initial data starting at $Z(0)$ will evolve towards $A$ or $B$ following the arrows $Z(\tau)$; data near the threshold will be marginally attracted towards the critical solution $Z^{\star}$. The points exactly on the critical manifold will be attracted to the critical solution.

2.2.2. Type I critical phenomena. Type I critical phenomena are the ones in which the blackhole formation turns on at finite mass and the critical solution presents a non-self-similar stationary or periodic solution configuration. The scaling quantity here is the lifetime of the metastable solution

$$
t_{p}=-\frac{1}{\lambda} \ln \left|P-P^{\star}\right|+\text { const },
$$

where $\lambda$ does not depend on the initial data. This scaling can be justified using simple arguments similar to the ones presented in [13] for the mass scaling in the type II case.

\section{Numerical setup}

In what follows we briefly describe the numerical setup used in the simulations and the procedure followed in the construction of the initial data. In essence, we use the Whisky2D code described in detail in [19] based on the three-dimensional code Whisky [20-22], to solve numerically and in two spatial dimensions the full set of Einstein equations

$$
G_{\mu \nu}=8 \pi T_{\mu \nu},
$$

where $G_{\mu \nu}$ is the Einstein tensor and $T_{\mu \nu}$ is the stress-energy tensor. More specifically, we evolve a conformal-traceless ' $3+1$ ' formulation of the Einstein equations as presented in [23], in which the spacetime is decomposed into 3D spacelike slices, described by a metric $\gamma_{i j}$, its embedding in the full spacetime, specified by the extrinsic curvature $K_{i j}$, and the gauge functions $\alpha$ (lapse) and $\beta^{i}$ (shift), which specify a coordinate frame. Axisymmetry is imposed using the "cartoon' technique [24] and the equation are solved using finite differencing of order 3 . The chosen slicing condition is the popular ' $1+\log$ ', while the chosen spatial gauge is the 
Gamma-freezing one. The field equations for the three-metric $\gamma_{i j}$ and the second fundamental form $K_{i j}$ are coupled with the equations of motion of general relativistic hydrodynamics

$$
\nabla_{\mu}\left(\rho u^{\mu}\right)=0, \quad \nabla_{\nu} T^{\mu \nu}=0,
$$

where $\rho$ is the (rest) baryonic mass density, $u^{\mu}$ is the four-velocity of the fluid and $T^{\mu \nu}$ is the stress-energy tensor of a perfect fluid

$$
T_{v}^{\mu}=\rho H u^{\mu} u_{v}+p \delta^{\mu}{ }_{v} .
$$

Here, $H \equiv 1+\epsilon+p / \rho$ is the specific enthalpy, $p$ is the pressure, $\delta^{\mu}{ }_{v}$ is the Kronecker delta and $\epsilon$ is the specific internal energy so that $e=\rho(1+\epsilon)$ is the energy density in the rest frame of the fluid. These equations are closed using an ideal-gas EOS $p=(\Gamma-1) \rho \epsilon$, with adiabatic exponent $\Gamma=2$. The solution of relativistic hydrodynamics equations is obtained via a conservative formulation of equation (10) as discussed in [19] and the use of highresolution shock-capturing (HRSC) schemes with a piecewise parabolic method (PPM) for the reconstruction of the primitive variables. The time stepping is done with a third-order totalvariation diminishing the Runge-Kutta algorithm. Finally, the spatial discretization is done on a uniform grid having the resolution of either $h=0.1$ (medium resolution) or $h=0.08$ (high resolution). The outer boundary of the computational domain is set at $R=15$ and we have verified that the proximity of the outer boundary does not influence significantly the critical solution.

The equilibrium configuration curves in the $\left(\rho, M_{\mathrm{ADM}}\right)$ plane and the perturbative oscillation frequencies quoted in the text have been computed using two codes kindly provided to us by Yoshida and Eriguchi [25] and Chirenti and Rezolla [26].

\subsection{Initial data}

The initial data consist of a family of spherical stars having fixed baryonic mass

$$
M_{b}=1.5194 \equiv \bar{M}_{b},
$$

constructed using a polytropic EOS $p=K \rho^{\Gamma}$, with $\Gamma=2$. Each model is computed by fixing its central rest-mass density, $\rho_{c}$, while the value of $K$ is fixed after imposing condition (12). The reason for this choice is that we want to guarantee that all the models considered have, at least initially, the same baryonic mass to the precision in expression (12). Solutions with different baryonic mass, in fact, are effectively in different phase spaces and thus not useful when looking at a critical behaviour. Of course, different models will also be slightly different because the perturbations will slightly alter their mass-energy or because although $M_{b}$ is conserved to high precision by employing a conservative formulation of the equations, it is nevertheless not conserved to machine precision. All of these latter errors, however, are entirely resolution dependent and can, therefore, be singled out by considering simulations at different resolutions.

These initial models have been evolved under the sole effects of the perturbations induced by the truncation error. Besides depending on resolution (and converging away), the amplitude of these perturbations is difficult to measure as it depends on a number of different sources of error, such as the interpolation error of the one-dimensional initial data on the threedimensional Cartesian grid, or the treatment of low-density 'atmosphere' regions, which are not measurable directly. However, an indirect measure can be obtained by looking at a short evolution of a stable spherical star which, in the absence of any numerical error, would not exhibit any dynamics but which, in practice, oscillates under the effects of these perturbations [1, 19-21, 27-29]. The amplitude of the observed oscillations can be then interpreted as an indirect measure of the numerical perturbation. In particular, we can consider the value of the 
Table 1. Properties of some of the representative models considered and shown either in figure 2 or in figures 6 and 7. More specifically, $N_{1}$ and $S_{1}$ are the extremes of the range of central densities considered, $P_{1}$ is a largely subcritical model which expands to models $P_{2}-P_{4}$ as the resolution is increased, while $Q_{1}$ and $R_{1}$ represent the closest super- and subcritical approximation of the critical solution, respectively.

\begin{tabular}{lllllll}
\hline Point & $\rho_{c}$ & $K$ & $M_{\mathrm{ADM}}$ & $M_{b}$ & Subcritical & Supercritical \\
\hline$N_{1}$ & 0.00395000 & 71.77 & 1.3879 & 1.5194 & $\sqrt{ }$ & - \\
$P_{1}$ & 0.00459316 & 71.39 & 1.3832 & 1.5194 & $\sqrt{ }$ & - \\
$P_{2}$ & 0.00341517 & 72.23 & 1.3754 & 1.5077 & $\sqrt{ }$ & - \\
$P_{3}$ & 0.00378525 & 71.58 & 1.3788 & 1.5134 & $\sqrt{ }$ & - \\
$P_{4}$ & 0.00387685 & 71.61 & 1.3809 & 1.5161 & $\sqrt{ }$ & - \\
$Q_{1}$ & 0.00459322 & 71.39 & 1.3832 & 1.5194 & - & $\sqrt{ }$ \\
$R_{1}$ & 0.0059322 & 71.39 & 1.3832 & 1.5194 & $\sqrt{ }$ & - \\
$S_{1}$ & 0.00508840 & 71.95 & 1.3842 & 1.5194 & - & $\sqrt{ }$ \\
\hline
\end{tabular}

average velocity in the radial direction during the first iterations as an estimate of the amplitude of an equivalent velocity perturbation. In this case, for a spherical star with $\rho_{c}=0.00128$ and $K=100$ evolved for 100 timesteps on an $h=0.1$ grid, we measure an average velocity $v^{r} \simeq 1.1 \times 10^{-5}$. Further insight can also be gained by the average of the momentum constraint violation in the radial direction and the Hamiltonian constraint violation, which we measure to be $\simeq 2.3 \times 10^{-7}$ and $\simeq 6.1 \times 10^{-6}$, respectively.

The determination of the critical value of the central density $\rho_{c}^{\star}$ is obtained rather straightforwardly via a bisection-like strategy within the initial interval

$$
0.00395<\rho_{c}<0.0050884
$$

where the extrema correspond to a stable oscillating star or to one collapsing promptly to a black hole, respectively.

The main properties of the initial data are collected in table 1 and summarized in figure 2, which reports the position of some of the most important models discussed in this paper in the $\left(\rho_{c}, M_{\mathrm{ADM}}\right)$ plane. More specifically, $N_{1}$ and $S_{1}$ are the extremes of the range of central densities considered (cf equation (13)), $P_{1}$ is a largely subcritical model which expands to models $P_{2}-P_{4}$ as the resolution is increased (cf figure 6), while $Q_{1}$ and $R_{1}$ represent the closest super- and subcritical approximation of the critical solution, respectively. Note that $R_{1}$ and $Q_{1}$ differ only by $4.6 \times 10^{-8} \%$ in the central density and thus they appear identical in the figure. Note also that $P_{1}, Q_{1}$ and $R_{1}$ are all on the unstable branch of the models of equilibrium and are therefore linearly unstable.

As a final remark we note that although the use of an axisymmetric system of equations is not strictly necessary for the spherically symmetric initial data considered here, their numerical solutions in two spatial dimensions via the Whisky2D code have been useful in view of the connections between the critical behaviour discussed here and the one presented in the companion paper [2], where the head-on collision of equal-mass NR is considered. The possibility of using the same numerical infrastructure and comparable truncation errors has been in fact very important in determining the connections between the two critical behaviours.

\section{Results}

In what follows we discuss the nonlinear dynamics of the spherical stars as these evolve away from their initial state on the unstable branch and exhibit a critical behaviour. 


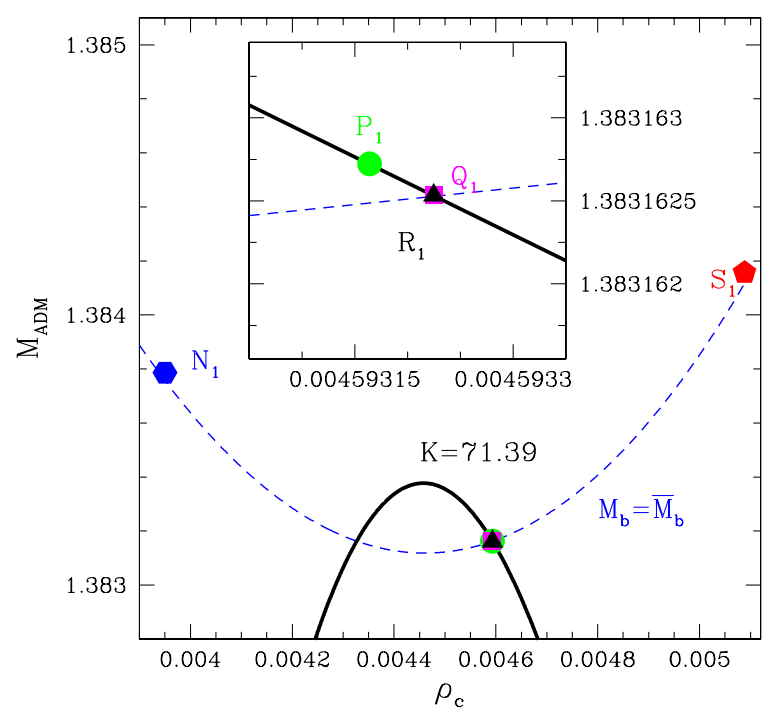

Figure 2. Position of some of the most important models in the $\left(\rho_{c}, M_{\mathrm{ADM}}\right)$ plane, where the solid (black) line refers to a sequence with $K=71.39$, while the dashed (blue) line refers to a sequence of models having the baryonic mass $M_{b}=1.5194=\bar{M}_{b}$. The points $N_{1}$ and $S_{1}$ are the extremes of the range of central densities considered (cf equation (13)), $P_{1}$ is a largely subcritical model, while $Q_{1}$ and $R_{1}$ represent the closest super- and subcritical approximation of the critical solution, respectively. The inset shows a magnification of the region near the critical solution; the properties of the model are reported in table 1 .

\subsection{Critical solution}

We first consider the evolution of models in the window (13) under the sole effect of the numerically induced perturbations. Some of these models, namely the supercritical ones, collapse to black hole, while others, namely the subcritical ones, undergo a sudden expansion followed by a relaxation towards the corresponding model on the stable branch of the spherical star solutions. This is clearly shown in figure 3 , which reports the evolution of the central rest-mass density and where different lines refer to different initial data in the interval

$$
0.0045931640625 \leqslant \rho_{c} \leqslant 0.00459371875 \text {. }
$$

By looking at the left panel of figure 3, it is quite apparent how the survival time of the metastable solution increases as the initial models approach the critical threshold and both the subcritical and the supercritical solutions overlap for a long part of the evolution, before departing exponentially. It is also worth remarking that the linear stability analyses of these models indicate that they are linearly unstable with a characteristic collapse time (i.e. the inverse of the imaginary part of the complex eigenfrequency of the fundamental mode) $\tau \simeq 440$. Yet, as shown in figure 3, the metastable models survive for much longer times and for almost $\tau \simeq 850$ for the models closest to the critical threshold.

A similar behaviour in the evolution of the central rest-mass density has been observed also in the simulations reported in [1], although those refer to magnetized and rotating stellar models and thus, being the result of three-dimensional simulations, are restricted to a much smaller interval of significant figures. In addition, and as mentioned in the introduction, evidence for a type I critical behaviour for the evolution of the central rest-mass density has been shown also in the head-on collision of two equal-mass spherical stars [10] and will be further discussed in the companion paper [2]. 

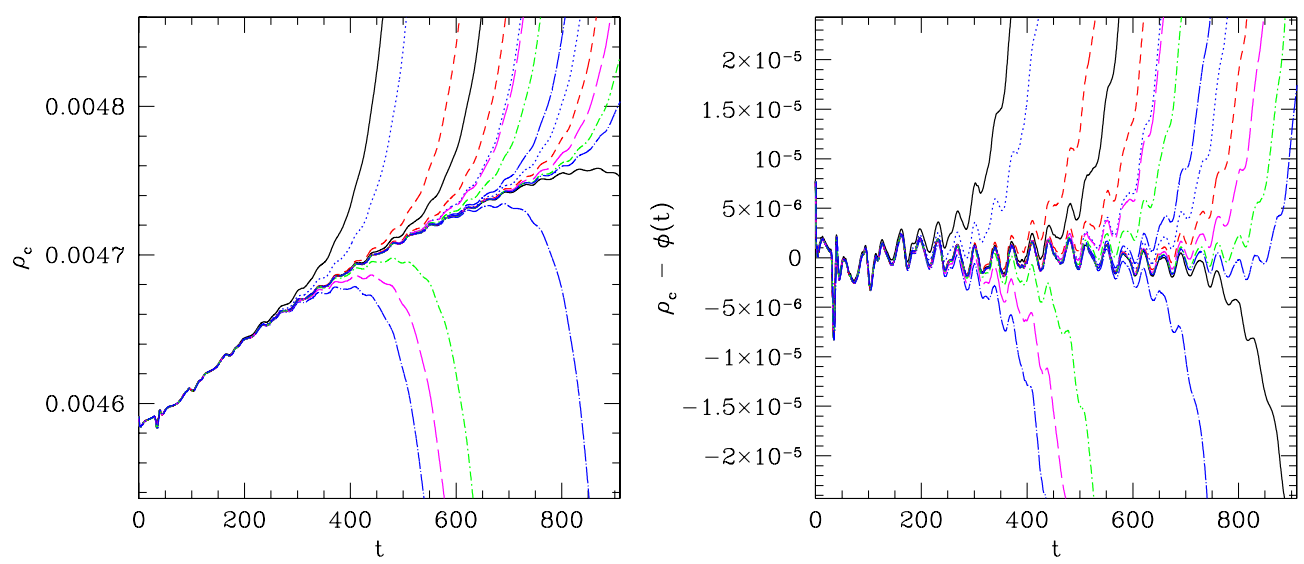

Figure 3. Left panel: evolution of the central rest-mass density near the critical threshold with different lines referring to different initial models. Right panel: the same as in the left panel but corrected for the secular evolution given by equation (15).

As the secular evolution in the central density is a well-known 'feature' of the numerical solution of relativistic multi-dimensional stellar models and has been observed in codes implementing very different numerical methods and formulations of the Einstein equations [1, 19-21, 27-29], we have isolated this secular behaviour by computing a least-squares fit of the common part of the evolution in order to isolate the true dynamics from the low-frequency numerical components. More specifically, we have modelled the evolution of the central rest-mass density of the metastable equilibrium via the Ansatz

$$
\phi(t)=\rho_{0}+\rho_{1} t+\rho_{2} \cos \left(2 \pi h_{1} t+\varphi_{1}\right)+\rho_{3} \cos \left(2 \pi h_{2} t+\varphi_{2}\right),
$$

where $\rho_{0}-\rho_{2}$ are just coefficients in the interpolation and do not have a particular physical meaning. On the other hand, the frequencies $h_{1}$ and $h_{2}$ are chosen as the two smallest frequencies appearing in the Fourier spectrum of the central density during the metastable phase (cf figure 4 and see also the discussion below on the spectral power density of the putative critical solution). The residuals after the fit are shown in the right panel of figure 3 and help considerably in appreciating the dynamics of the unstable models near the critical value.

Using a large set of simulations with the resolution of $h=0.1$ and a straightforward bisection strategy, we have located the critical threshold to black-hole formation at a central density

$$
\rho_{c}^{\star}=0.004593224802 \pm 2.1 \times 10^{-12} .
$$

Clearly, we expect this value to depend on the initial perturbation and thus on the resolution used, as well as on the numerical method employed. On the other hand, we also expect the associated solution and the critical exponent to be 'universal', in the sense that they should not depend sensitively on the perturbation or on the particular family of initial data as far as this family is characterized by a single parameter and thus intersects the critical manifold $\mathcal{C}$ in a single point which is near enough to this solution. In this case, in fact, the associated critical solution is supposed to be at least locally attractive on a sub-manifold of the phase space of codimension 1.

To validate that the behaviour discussed so far and shown in figure 3 does represent a type I critical behaviour, we compute the survival time of the metastable solution $\tau$, i.e. the 'escape time', and study how this varies as the critical solution is approached. We recall 


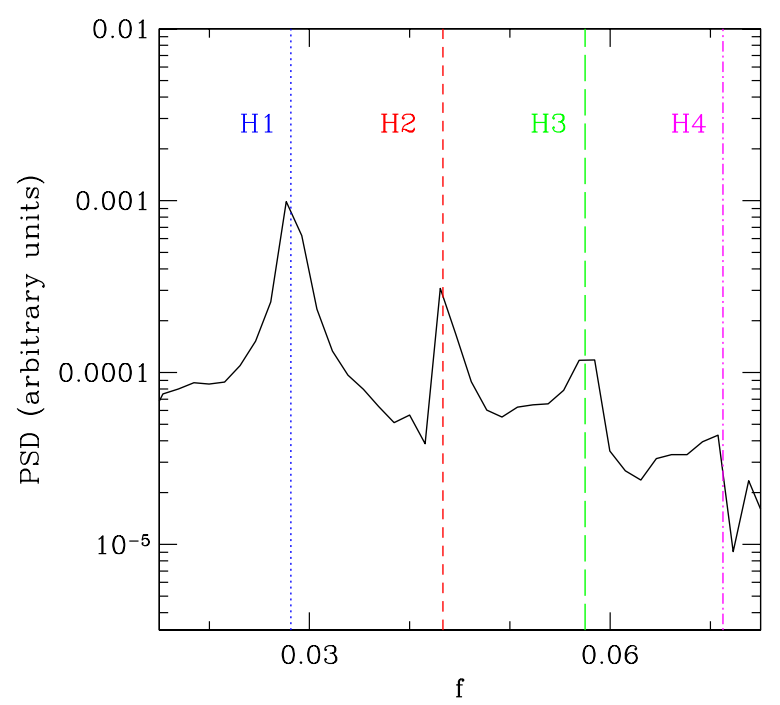

Figure 4. Power spectral density of the evolution of the central rest-mass density for the model closest to the putative critical solution (i.e. with $\rho_{c}=0.0045932248034$ ) when the secular drift part (15) has been removed from the data. The eigenfrequencies associated with the corresponding spherical star model are also shown as vertical lines.

that we expect that the escape time near the critical for a type I critical phenomena should behave as

$$
\tau=-\frac{1}{\lambda} \ln \left|\rho_{c}-\rho_{c}^{\star}\right|+\text { const }
$$

and such an expected solution is indeed shown as a dashed line in figure 5. Also shown with squares and triangles are the computed escape times for different initial data and different resolutions (blue squares for $h=0.1$ and red triangles for $h=0.08$ ). The latter are calculated in terms of the time $\tau_{\epsilon}$ at which the relative difference between the observed central baryonic density and the best approximation of the critical solution $\phi(t)$ (equation (15)) become larger than $\epsilon$. We find that, for a large enough $\epsilon$, such that $1 \gg \epsilon>\epsilon^{\star}>0$, these times depend only weakly on $\epsilon$ and thus give a good measure of the departure time from the critical solution. A value of $\epsilon=0.5 \%$ provides a sufficiently accurate measure and this is the one employed for the data points shown in figure 5 . We finally estimate the critical exponent $\lambda$ by making a linear least-squares regression of the data points of sub- and supercritical solutions and then by taking the average of the two values. Using the medium resolution $h=0.1$ simulations, we therefore obtain for the critical exponent,

$$
\lambda=0.02149665,
$$

with a coefficient of determination $R^{2}$ relative to the linear regression (17) and computed on the full dataset containing both sub- and supercritical solutions, of 0.960517 . The critical exponent (18) is found also in the case of the $h=0.08$ simulations, although in this case the scattering is somewhat larger and the data agree within $7 \%$. We note that these high-resolution simulations are computationally very expensive and this is why we have restricted them to a smaller set of initial data. Clearly, the match between the computed escape times and the one expected from the critical behaviour is very good over the six orders of magnitude in $\left|\rho_{c}-\rho_{c}^{\star}\right|$ spanned by our dataset and thus provides convincing evidence that indeed critical behaviour can be found in the dynamics of linearly unstable spherical stars. 

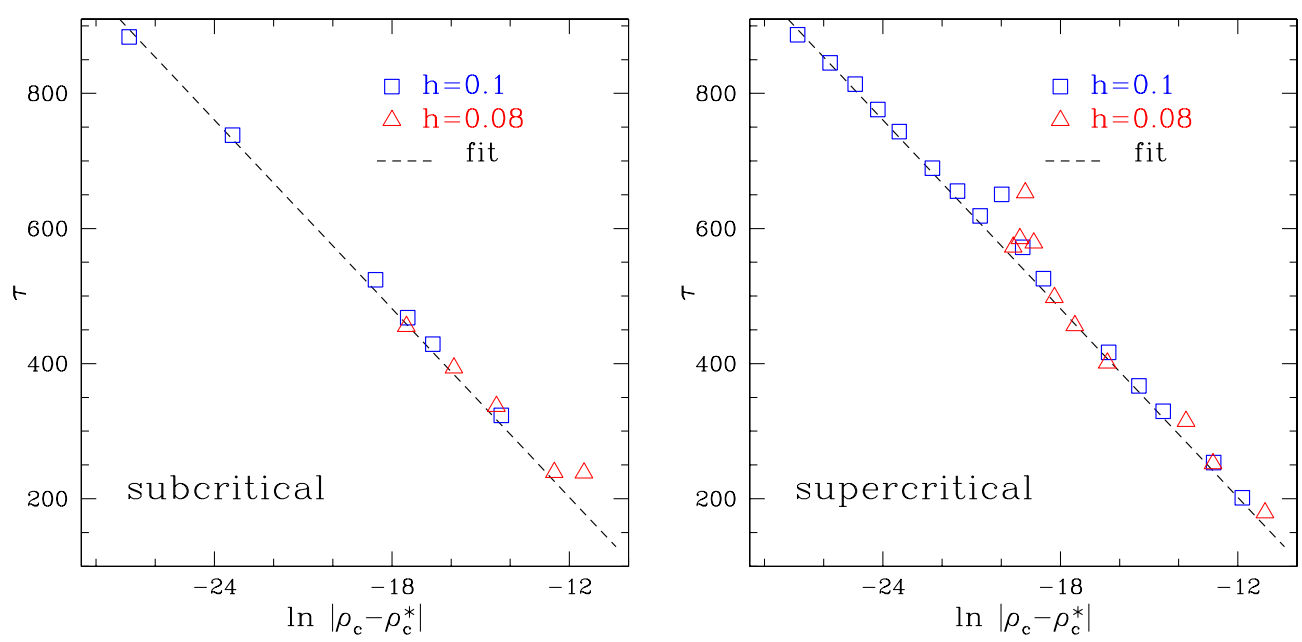

Figure 5. Escape time $\tau$ as a function of $\ln \left|\rho-\rho^{\star}\right|$ for subcritical (left panel) and supercritical solutions (right panel), respectively. The blue squares correspond to the results obtained with the $h=0.1$ resolution, while the red triangles to the results obtained with the $h=0.08$ resolution. The dashed lines represent the fit obtained using equation (17) with $\lambda$ obtained from the $h=0.1$ solutions.

As a final remark we note that while the evidence for a critical behaviour is clear, much less clear is the physics of the critical solution which is, after all, a perturbed spherical star. Recent studies of nonlinear perturbations of relativistic spherical stars have shown that linearly unstable stars can be stabilized via nonlinear couplings among higher order modes [30]. It is possible that such a nonlinear coupling is present also here and we conjecture therefore that the stability of the metastable solution is due to the mode coupling of the first overtones of the fundamental mode. A support to this conjecture comes from the power spectral density in figure 4, which shows that, apart from the F-mode which is obviously missing as it has only imaginary eigenfrequency, the spectrum of the metastable solution is essentially identical to the one of an excited spherical star with $\left(\rho_{c}, K\right)=\left(\rho^{\star}, K^{\star}\right)$ and $M_{b}=\bar{M}_{b}$. Interestingly, most of the energy is in the first overtone, $H 1$, even though the numerical perturbation can be thought as 'white noise' exciting all the modes of the star with almost equal energy. The behaviour discussed above persists also when considering models with higher spatial resolutions.

\subsection{Subcritical solutions}

While the final fate of supercritical solutions is clearly that of leading to a collapse and to the formation of a black hole, one of the subcritical solutions deserves a more detailed explanation. As one would expect, given that the initial data represent linearly unstable stars, the subcritical solutions show a first expansion as the star migrates to the stable branch of the equilibrium configurations, which is then followed by a slow relaxation where the central rest-mass density exhibits strong oscillations around smaller and smaller values, which would eventually reach in the continuum limit, the value corresponding to the model on the stable branch having the same gravitational mass of the initial one. In practice, however, the migration to the stable branch is accompanied by small losses both in the gravitational mass and in the rest mass which, although smaller than $\simeq 0.7 \%$, needs to be taken properly into account.

More specifically, we have analysed in detail the evolution of the largely subcritical model $P_{1}$ (cf table 1), which is an unstable spherical star with an F-mode whose imaginary part of 


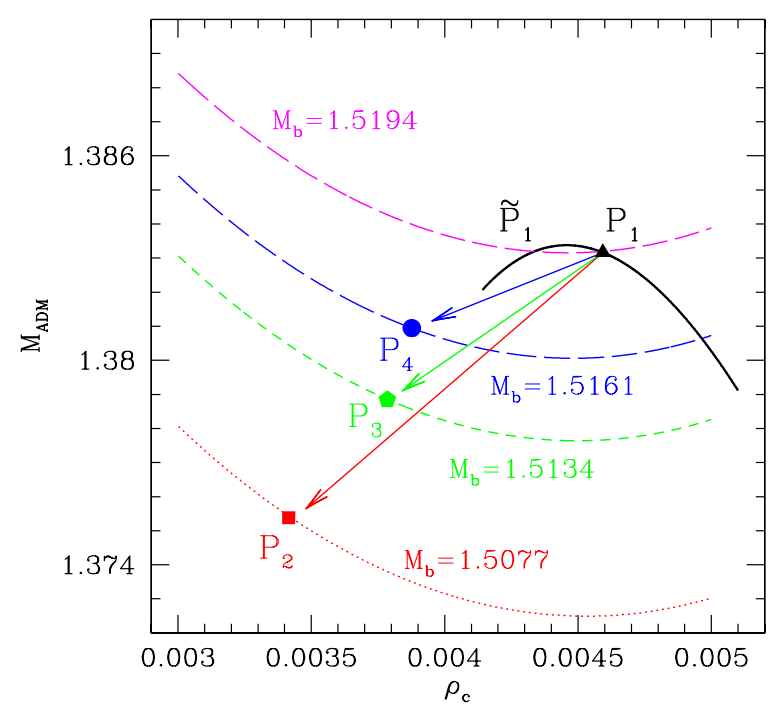

Figure 6. Dynamics of the migration on a $\left(\rho_{c}, M_{\mathrm{ADM}}\right)$ plane. The linearly unstable and largely subcritical model $P_{1}$ migrates to a new solution on the stable branch of equilibrium configurations. Indicated with $P_{2}-P_{4}$ are the new asymptotic states for resolutions $h=0.1-0.08$, respectively. Indicated with a thick solid line is the sequence of initial models having the same polytropic index of $P_{1}$, while indicated with dotted and dashed lines are sequences of models having the same rest mass as the asymptotic models $P_{2}-P_{4}$. Finally, shown as $\tilde{P}_{1}$ is the asymptotic state of $P_{1}$ in the continuum limit; note that even for the coarse-resolution case the changes in baryonic and rest mass are only of $\simeq 0.7 \%$.

the eigenfrequency is $v_{i}=0.461 \mathrm{kHz}$. We therefore evolved such a model with three different spatial resolutions of $h=0.1, h=0.09$ and $h=0.08$, and studied its migration to the stable branch. The asymptotic state of the solution and in particular to the final central rest-mass density $\rho_{f}$ is estimated by modelling the time evolution of the oscillating star on the stable branch with a simple Ansatz of the type $\rho(t)=\rho_{f}+\rho_{1} / t$ and by performing a nonlinear least-squares fit on an appropriate window including the final part of the dynamics. For any given resolution we have then computed the total baryonic-mass losses due to the numerical dissipation $\Delta M_{b}=M_{b}-M_{b, f}$, and determined the polytropic coefficient $K_{f}$ yielding a spherical stellar model with the central rest-mass density $\rho_{f}$ and the baryon mass $M_{b, f}$. Clearly, for such a model it is then also possible to compute the gravitational mass and thus track the migration on a $\left(\rho_{c}, M_{\mathrm{ADM}}\right)$ plane.

The overall results of these migrations are shown in figure 6 , where we report the stellar configurations on $M_{b}=$ const curves. The minimum of each curve corresponds to the maximum in the usual $\left(\rho, M_{\mathrm{ADM}}\right), K=$ const, plots and separates the stable and unstable branches of solutions. When a resolution of $h=0.1$ is used, model $P_{1}$ migrates to the new asymptotic model $P_{2}$, while it will migrate to models $P_{3}$ and $P_{4}$ as higher resolutions of $h=0.09$ and $h=0.08$ are used, respectively. Note that already with the coarsest resolution of $h=0.1$, the losses in gravitational masses are $\simeq 0.65 \%$ and that these decrease to $\simeq 0.16 \%$ when a resolution of $h=0.08$ is used. Finally, indicated with $\tilde{P}_{1}$ is the expected asymptotic model when the numerical losses are extrapolated to the continuum limit ${ }^{3}$; clearly, in the limit

\footnotetext{
${ }^{3}$ Note that we do not mark this point with a symbol as it does not correspond to a numerically computed value, as
} instead for $P_{2}, P_{3}$ and $P_{4}$. 
$h \rightarrow 0$, the migration of model $P_{1}$ takes place to a new state having the same gravitational and baryonic mass as the initial one.

\subsection{Perturbation of nearly critical solutions}

As discussed in section 4.1, the central rest-mass density of the linearly unstable models can be used as a critical parameter for the gravitational collapse of a linearly unstable spherical star, in contrast to what has been observed for example by Novak in [7] or by Noble and Choptuik in [9]. We believe that this is due to the very different set of initial data selected here and in $[7,9]$. Indeed, the reason why this behaviour has not been observed in many previous studies is that we consider initial stellar models that are already linearly unstable, in contrast with what done in [7,9], where the initial models are instead linearly stable and then subject to a perturbation (either by introducing a radial velocity [7, 9], or by considering employing the interaction with a scalar field [8]). For our set of initial data, therefore, the critical solution is essentially a spherical star with an unstable F-mode, and any finite perturbation exciting this mode will change the solution in a dramatic way. (A discussion of this change within a phase-space description will be made later on when presenting figure 8. $)^{4}$

To confirm this hypothesis, we follow [7,9], and construct a new family of spherical initial data obtained by perturbing the slightly supercritical model $Q_{1}$ (cf table 1 ) via the addition of a radial velocity perturbation in the form of the three-velocity component

$$
v^{r}(x)=\frac{U}{2}\left(3 x-x^{3}\right), \quad x \equiv \frac{r}{R_{\star}},
$$

where $U$ is the amplitude of the perturbation at the surface of the star, $R_{\star}$, and can be either positive (outgoing radial velocity) or negative (ingoing radial velocity). Because perturbation (19) matches the eigenfunction of an idealized F-mode perturbation, it should excite the only unstable mode of the critical solution.

Performing simulations for different values of $U$ and a resolution $h=0.1$ we find, not surprisingly, that for negative values of $U$ the perturbed models of $Q_{1}$ collapse to a black hole. Furthermore, because in this case the radial velocity accelerates the development of the unstable mode, the larger the values of $U$ the shorter the time to collapse, i.e. $\tau \sim-c_{1} \log (U)+c_{2}$, where $c_{1}$ and $c_{2}$ are positive constant coefficients. On the other hand, for positive values of $U$, the perturbed models of $Q_{1}$, which we recall are supercritical for $U=0$, become subcritical and show the same qualitative behaviour as that of model $R_{1}$. Hence, a suitably perturbed supercritical model can behave as a subcritical one.

The dynamics of these perturbed, nearly critical models is shown in figure 7 , where the solid (black) line represents the supercritical solution $Q_{1}$, while the dotted (blue) line represents the subcritical solution $R_{1}$. The dashed lines show again the evolution of $Q_{1}$, but when subject to a positive (red short-dashed line) or negative (green long-dashed line) velocity perturbation. The dynamics shown in figure 7 underlines an important characteristic of critical phenomena: the precise value of the critical parameter at the intersection between the one-parameter family of solutions and the critical manifold depends on the family itself. In particular, this means that if we fix a value of the perturbation amplitude, $U \neq 0$, we expect to find the critical solution at a value of $\rho_{c}^{\star}(U)$ different from the one quoted in (16) which is attained in the case $U=0$. For this reason the application of a non-infinitesimal perturbation to a nearly critical solution results in a dramatic change in the dynamics of the system.

\footnotetext{
4 With 'perturbation' we are here referring to a globally coherent, resolution-independent perturbation such as the one given in equation (19). This has to be contrasted with the random, truncation-error-induced and resolution-dependent perturbations we have considered in section 4.1 .
} 


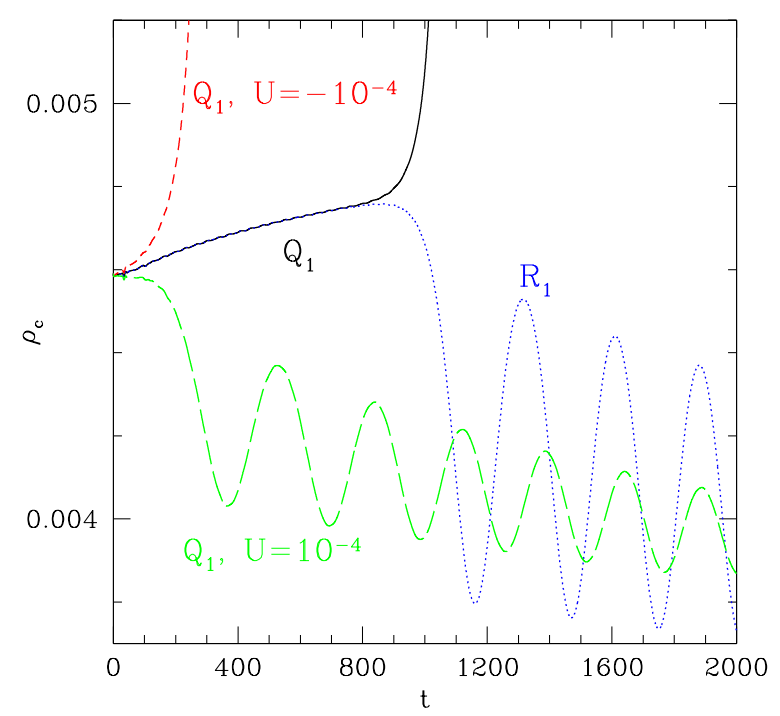

Figure 7. Perturbation of nearly critical solutions. The solid (black) line represents the supercritical solution $Q_{1}$, while the dotted (blue) line represents the subcritical solution $R_{1}$. The dashed lines show again the evolution of $Q_{1}$, but when subject to a negative (red short-dashed line) or positive (green long-dashed line) velocity perturbation. Clearly, in the latter case the supercritical solution $Q_{1}$ becomes subcritical and shows the same behaviour as the solution $R_{1}$.

The phase-space representation of this concept is summarized in figure 8 , where we show two one-parameter families of perturbed TOV initial data, whose critical parameter, $\rho_{c}$, is the central rest-mass density. The perturbation is given by the composition of truncation errors and of a radial velocity perturbation $U$ in the form (19), where $U=0$ or $U=U_{0}>0$. As these families represent different initial configurations, they will intersect the critical manifold $\mathcal{C}$ at two different points, with correspondingly different values of the critical parameter $\left\{0, \rho_{c}^{*}(0)\right\}$ and $\left\{U_{0}, \rho_{c}^{*}\left(U_{0}\right)\right\}$ (these points are marked as filled circles) ${ }^{5}$. In particular, when $U$ runs between 0 and $U_{0}$, the set of critical configurations $\left\{U, \rho_{c}^{*}(U)\right\}$ will represent a curve on the critical manifold $\mathcal{C}$ and this is shown with a violet solid line in figure 8 . Considering now a configuration near $\left\{0, \rho_{c}^{*}(0)\right\}$ and applying to it a velocity perturbation in the form (19) with $U=U_{0}$ will produce a new configuration $\left\{U_{0}, \rho_{c}^{*}(0)\right\}$ which is not necessarily on the critical manifold (this is marked with a filled square). Indeed, the whole family $\left\{U, \rho_{c}^{*}(0)\right\}$, that is the set of configurations with a nonzero initial velocity perturbation but central density which is the critical one for the zero-velocity case, is in general expected to be outside the critical domain. The family $\left\{U, \rho_{c}^{*}(0)\right\}$ is shown with a black dot-dashed line in figure 8 .

As a final remark we note that another important difference between the work presented here and that in $[7,9]$ is that we find evidence of a type I critical behaviour with a periodic solution, in contrast to what found in [7, 9], which is instead of type II and with DSS solutions. We believe that the origin of this important difference and of the presence of a periodic solution is in our use of an ideal-fluid EOS and hence in the presence of an overall scale in the problem. Conversely, the spherical stars considered in the above-mentioned works were evolved using either an ultra-relativistic EOS [5] (which, as commented in the Introduction, are intrinsically

\footnotetext{
5 In our notation, the point $\left\{U_{0}, \rho_{c}^{*}\left(U_{0}\right)\right\}$ is the critical solution with initial velocity perturbation given by equation (19) with $U=U_{0}$. Similarly, a configuration $\left\{U_{0}, \rho_{c}^{*}(0)\right\}$ will be a member of the family with initial velocity perturbation $U_{0}$, but with a central density which is the critical one for a model with $U=0$.
} 


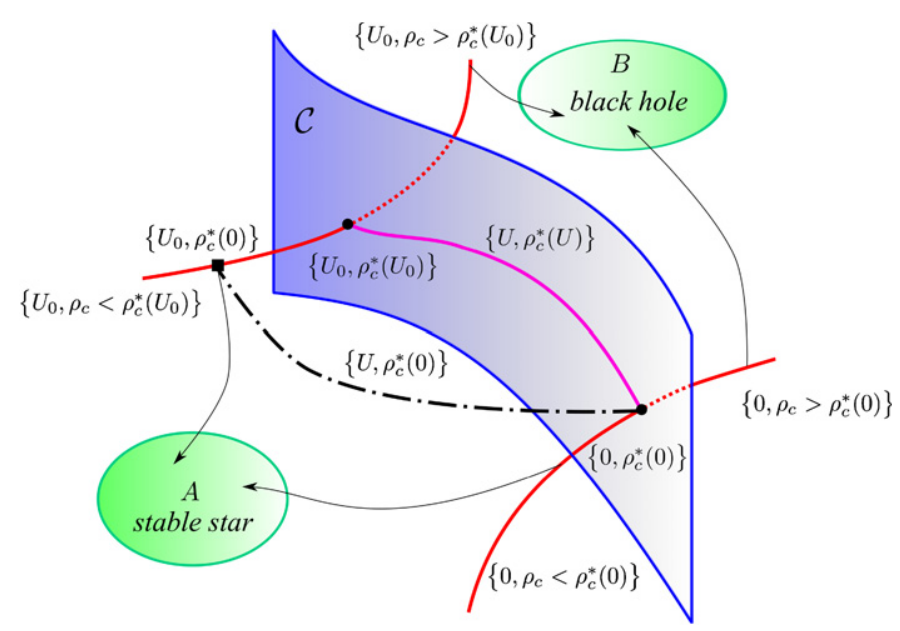

Figure 8. Phase-space diagram representation of nearly critical solutions. In particular, we show with red solid curves two one-parameter families of initial data, perturbed by the addition of a radial velocity profile in the form (19) with $U=0$ or $U=U_{0}>0$. The locus of the critical points, $\left\{U, \rho_{c}^{*}(U)\right\}$, is shown with a violet solid line, while the family of initial data $\left\{U, \rho_{c}^{*}(0)\right\}$ is shown with a black dot-dashed line and the point $\left\{U_{0}, \rho_{c}^{*}(0)\right\}$ is marked with a filled square. The latter represents therefore the family of initial data obtained by adding a velocity perturbation with amplitude $U$ to the model with central density would when $U=0$. Also highlighted with filled circles are the critical points for the families with $U=0$ and $U=U_{0}$, i.e. $\left\{0, \rho_{c}^{*}(0)\right\}$ and $\left\{U_{0}, \rho_{c}^{*}\left(U_{0}\right)\right\}$.

scale-free) or with very strong perturbations [7, 9], thus in a regime of the EOS which is approximatively ultra-relativistic [13].

\section{Conclusions}

In general, critical phenomena in gravitational collapse are of great interest because they play a central role in the phase transition of the families of solutions in general relativity. In a more specific context of the dynamics of NSs, type I critical phenomena have seen a renewed interest when it was shown that a critical behaviour of this type is produced in the head-on collision of NSs [10] or in the dynamics of rotating magnetized stars [1]. With the goal of studying in more detail the occurrence of type I critical collapse in NSs, we have therefore employed the 2D general relativistic code Whisky2D to study a large set of spherical stellar models having a constant baryon mass. Differently from what done before by other authors, e.g. [7, 9], we have considered stellar models that are on the 'right' branch of the models of equilibrium and thus linearly unstable.

Using a simple ideal-fluid EOS and very small perturbations which are entirely induced by the truncation error, we have found that our family of initial data exhibits a clear type I critical behaviour at a threshold central rests-mass density of $\rho_{c}^{\star}=0.004593224802 \pm 2.1 \times 10^{-12}$ and with a critical exponent $\lambda=0.02149665$. These results thus confirm the conclusions reached by Liebling et al [1] but also provide a more quantitative determination of the threshold and of the nature of the critical scaling. Exploiting in fact the relative simplicity of our system, we were able carry out a more in-depth study providing solid evidence of the criticality of this phenomenon and also to give a simple interpretation of the putative critical solution as a spherical solution with the unstable mode being the fundamental F-mode. As a result, we 
have shown that for any choice of the polytropic constant, the critical solution distinguishes the set of subcritical models migrating to the stable branch of the models of equilibrium from the set of supercritical models collapsing to a black hole.

Furthermore, we have studied how the dynamics changes when the numerically perturbation is replaced by a finite-size, resolution-independent velocity perturbation and show that in such cases a nearly critical solution can be changed into either a sub- or a supercritical one. Finally, the work presented here is of direct help in understanding why the critical behaviour shown in the head-on collision of two NR is indeed of type I and why it can be explained simply in terms of the creation of a metastable stellar model on the unstable branch of equilibrium solutions [2].

\section{Acknowledgments}

It is a pleasure to thank Bruno Giacomazzo and Filippo Galeazzi for their help and assistance with the Whisky2D code. We are also grateful to Giulio Magli and Carsten Gundlach for useful discussions and Shin'ichiro Yoshida and Cecilia Chirenti for providing the codes used to compute the spherical static models and their linear radial oscillation frequencies. This work was supported in part by the IMPRS on 'Gravitational-Wave Astronomy', by the DFG grant SFB/Transregio 7 and by 'CompStar', a Research Networking Programme of the European Science Foundation. The computations were performed on the Damiana cluster at the AEI.

\section{References}

[1] Liebling S L, Lehner L, Neilsen D and Palenzuela C 2010 arXiv:1001.0575v1

[2] Kellerman T, Rezzolla L and Radice D 2010 Class. Quantum Grav. 27235016

[3] Choptuik M W 1993 Phys. Rev. Lett. 709

[4] Evans C R and Coleman J S 1994 Phys. Rev. Lett. 72 1782-5

[5] Hara T, Koike T and Adachi S 1996 arXiv:gr-qc/9607010

[6] Neilsen D W and Choptuik M W 2000 Class. Quantum Grav. 17761

[7] Novak J 2001 Astron. Astrophys. 376606 (arXiv:gr-qc/0107045v1)

[8] Noble S C 2003 A numerical study of relativistic fluid collapse PhD Thesis University of Texas at Austin (arXiv:gr-qc/0310116v1)

[9] Noble S C and Choptuik M W 2008 Phys. Rev. D 78064059 (arXiv:0709.3527)

[10] Jin K J et al 2007 Phys. Rev. Lett. 98131101

[11] Musco I, Miller J C and Rezzolla L 2005 Class. Quantum Grav. 22 1405-24

[12] Musco I, Miller J C and Polnarev A G 2009 Class. Quantum Grav. 26235001 (arXiv:0811.1452)

[13] Gundlach C and Martín-García J 2007 Living Rev. Rel. 105

[14] Brady P, Choptuik M, Gundlach C and Neilsen D 2002 Class. Quantum Grav. 19 6359-75

[15] Cahill M E and McVittie G 1970 J. Math. Phys. 21382

[16] Wan M B, Jin K J and Suen W M 2008 arXiv:0807.1710v2

[17] Gundlach C 1999 Living Rev. Rel. 24

[18] Gundlach C 1997 Phys. Rev. D 55695 (arXiv:gr-qc/9604019)

[19] Kellerman T, Baiotti L, Giacomazzo B and Rezzolla L 2008 Class. Quantum Grav. 25225007 (arXiv:0811.0938)

[20] Baiotti L, Hawke I, Montero P J, Löffler F, Rezzolla L, Stergioulas N, Font J A and Seidel E 2005 Phys. Rev. D 71024035 (arXiv:gr-qc/0403029)

[21] Giacomazzo B and Rezzolla L 2007 Class. Quantum Grav. 24 S235 (arXiv:gr-qc/0701109)

[22] Baiotti L, Giacomazzo B and Rezzolla L 2008 Phys. Rev. D 78084033 (arXiv:0804.0594)

[23] Pollney D et al 2007 Phys. Rev. D 76124002 (arXiv:0707.2559)

[24] Alcubierre M, Brandt S R, Brügmann B, Holz D, Seidel E, Takahashi R and Thornburg J 2001 Int. J. Modern Phys. D 10 273-89 (arXiv:gr-qc/9908012)

[25] Yoshida S and Eriguchi Y 2001 Mon. Not. R. Astron. Soc. 322389

[26] Chirenti C B M H and Rezzolla L 2007 Class. Quantum Grav. 24 4191-206 (arXiv:0706.1513) 
[27] Font J A, Goodale T, Iyer S, Miller M, Rezzolla L, Seidel E, Stergioulas N, Suen W M and Tobias M 2002 Phys. Rev. D 65084024 (arXiv:gr-qc/0110047)

[28] Duez M D, Liu Y T, Shapiro S L and Stephens B C 2005 Phys. Rev. D 72024028

[29] Anderson M, Hirschmann E W, Lehner L, Liebling S L, Motl P M, Neilsen D, Palenzuela C and Tohline J E 2008 Phys. Rev. D 77024006 (arXiv:0708.2720)

[30] Gabler M, Sperhake U and Andersson N 2009 Phys. Rev. D 80064012 (arXiv:0906.3088) 